\title{
Grain-size distribution effects in plastic flow and failure
}

\author{
M.P. Phaniraj, M.J.N.V. Prasad, A.H. Chokshi* \\ Department of Metallurgy, Indian Institute of Science, Bangalore 560 012, India
}

\begin{abstract}
There has been considerable success over the past five decades in developing a phenomenological and micromechanism-based understanding of plastic flow, creep and superplasticity. Although it is widely known that grain sizes have a distribution in polycrystals and nanocrystals, this factor is usually not included in most analysis of deformation and failure. Experimental observations relating to the influence of grain size distributions are discussed briefly, and an analysis is developed to consider the influence of this factor on the transition from grain boundary strengthening to grain boundary weakening in nanocrystalline materials. The transition from grain boundary strengthening to weakening becomes broader with an increase in the standard deviation of the grain size distribution. It is demonstrated that the observed standard deviations for grain size distributions and nominal errors in grain size measurements can lead to substantially different experimental observations under nominally identical conditions.
\end{abstract}

Keywords: Grain size distribution; Diffusion creep; Hall-Petch strengthening; Nanocrystals

\section{Introduction}

The time dependent plastic deformation of materials, termed creep, is an important design consideration for structural applications at high temperatures. It has been well known for several decades now, that creep is influenced primarily by factors such as the stress $\sigma$, the absolute temperature $T$ and the microstructure of materials. The many important phenomenological studies on creep by Dorn and Mukherjee, have led to what is now considered a standard equation for high temperature plastic flow $[1,2]$

$\dot{\varepsilon}=\frac{A D G b}{k T}\left(\frac{b}{d}\right)^{p}\left(\frac{\sigma}{G}\right)^{n}$

where $\dot{\varepsilon}$ is the steady-state strain rate, $A$ is a dimensionless constant, $D$ is the appropriate diffusion coefficient, $G$ is the shear modulus, $b$ is the magnitude of the Burgers vector, $k$ is Boltzmann's constant, $d$ is the grain size, and $p$ and $n$ are constants termed the inverse grain size and stress exponent, respectively. The diffusion coefficient is given as $D=D_{0} \exp (-Q / R T)$, where $D_{0}$ is a frequency factor, $Q$ is the appropriate activation energy, and $R$ is the gas constant.

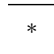

In general, creep may be influenced by many different microstructural parameters such as the grain size, stacking fault energy, and the dislocation density. Although the stochastic nature of microstructural features such as dislocation density, grain size distribution, grain boundary energies and grain boundary diffusion are known, these factors are not included explicitly in many creep rate equations. Thus, for example, most experimental studies use the linear intercept method to characterize the arithmetic mean linear intercept grain size, and use this value in Eq. (1).

The purpose of this paper is to examine the influence of grain size distribution on plastic flow and fracture. The next section describes some relevant experimental observations in superplastic deformation, creep cavitation, and geological materials. This is followed by an examination of experimental observations on grain size distribution in nanocrystals, an analysis of the effect this distribution on grain boundary strengthening and weakening, together with implications for experimental observations on hardness in nanometals.

\section{Experimental observations on effect of grain size distribution}

\subsection{Superplastic deformation}

Although grain boundary sliding, associated with diffusion creep or as an independent process, is accepted as the major 

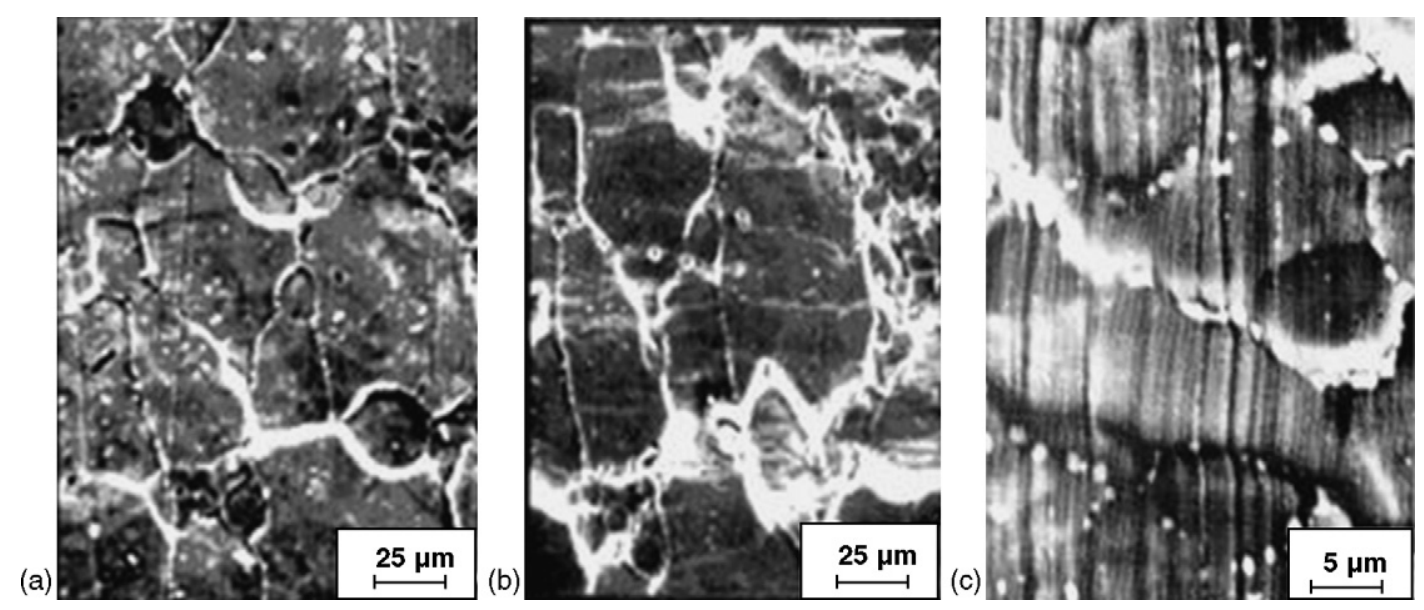

Fig. 1. Scanning electron micrographs of a superplastically deformed Al-Li alloy showing coarse grained regions at a strain of (a) 16\% and (b) $78 \%$, and a fine grained region at a strain of (c) $16 \%$ [5].

strain contribution process during superplastic deformation, the rate controlling mechanism has not yet been unambiguously identified, and plastic flow has been attributed variously to processes such as grain boundary sliding accommodated by diffusion creep or dislocation motion [3]. Raj and Ghosh [4] developed an interesting model for superplastic deformation which assumed that fine grains and coarse grains can deform by diffusion and intragranular dislocation creep processes, respectively. With a suitable choice of a bimodal grain size distribution, the model was able to reproduce the sigmoidal relationship between stress and strain rate that has been reported widely for superplastic alloys.

An experimental study on a superplastic $\mathrm{Al}-\mathrm{Li}$ alloy revealed that under some conditions, the alloy exhibited a bimodal grain size distribution, with coarse and fine grains having dimensions of $\sim 50$ and $10 \mu \mathrm{m}$, respectively [5]. Mechanical testing revealed that the alloy exhibited a typical sigmoidal relation between stress and strain rate, with an optimum strain rate sensitivity $m$ $(=1 / n)$ of $\sim 0.5$. Fig. $1 \mathrm{a}$ and $\mathrm{b}$ depicts scanning electron micrographs of the surfaces of polished specimens inscribed with marker lines, in a coarse grained region of a specimen at elongations of $\sim 16$ and $78 \%$, respectively. Inspection reveals that the initially straight marker lines on the surfaces are curved inside the grains and that the coarse grains become more elongated along the tensile axis. In contrast, Fig. 1c illustrates the retention of an equiaxed grain shape, the maintenance of straight marker lines within grains, and sharp offsets at grain boundaries indicating the occurrence of grain boundary sliding in the fine grained region. These experimental observations confirm the validity of assumptions in some models that fine and coarse grains within a specimen can deform by different mechanisms.

Recently Bai and Raj [6] extended the analysis to include a log-normal grain size distribution. Interestingly, this approach does not lead to a sigmoidal relationship between stress and strain rate. The analysis shows that the transition from intragranular dislocation creep $(n=5)$ to diffusion creep $(n=1)$ can occur over many decades of strain rates in materials with a high standard deviation of grain size distribution.

\subsection{Creep cavitation}

Sintering of alumina powders co-doped with magnesia and zirconia led to the development of a bimodal grain size distribution with coarse and fine grained regions having grain sizes of $\sim 10$ and $1 \mu \mathrm{m}$, respectively [7]. Examination of the polished tensile surface of a sample deformed by creep in 4-point bending mode indicated that cavitation was very inhomogeneous, Fig. 2a. Thermal etching of the samples revealed that cavitation occurred only in the coarse grained regions, as shown in Fig. $2 \mathrm{~b}$ from a region with a grain boundary crack.

Such observations can be rationalized in terms of load shedding from the fine grained to the coarse grained regions. Creep
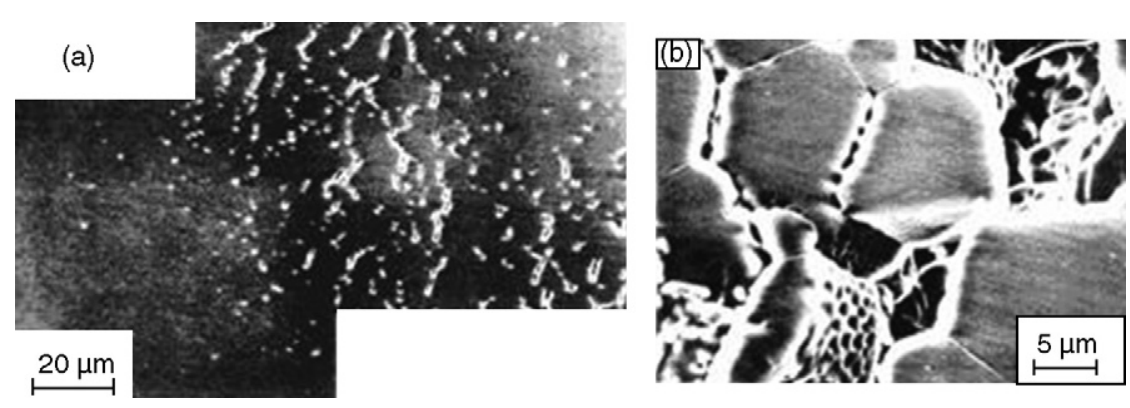

Fig. 2. Scanning electron micrographs of the tensile surface of a creep deformed polycrystalline alumina showing (a) inhomogeneous cavitation and (b) preferential cavitation in coarse grained regions [7]. 
in alumina is usually attributed to some form of diffusion or interface-reaction controlled diffusion creep, under which conditions

$\dot{\varepsilon} \propto \frac{\sigma^{n}}{d^{p}}$

where $n \sim 1$ to 2 , and $p \sim 2$ to 3 . Thus, under iso-strain rate conditions, an increase in grain size will lead to an increase in stress, which enables cavitation in the coarse grained regions.

\subsection{Geological materials}

In an interesting experimental study on high temperature deformation on an equi-volume two phase mixture of calcite and anhydrite, Heilbronner and Bruhn [8] found that samples with an essentially identical two-dimension grain size exhibited substantially different flow stress. The differences were attributed to variations in the three-dimensional grain size distribution.

Ter Heege et al. [9] developed a model for creep in materials with a log-normal grain size distribution deforming by diffusion creep and dislocation creep, using iso-strain rate or iso-stress assumptions. The model was applied successfully to experimental results on olivine, and it was demonstrated that the median and width of the grain size distribution plays an important role in creep; this has important implications for modeling geological deformation in the crust and mantle.

\section{Influence of grain size distribution on deformation in nanocrystalline materials}

For the remainder of the paper, for the sake of clarity, the grain size will be denoted as $L$ rather than $d$. It has been known for many decades now, that the change in crystallographic orientation across grain boundaries can lead to strengthening, and this is given by the Hall-Petch equation [10,11]:

$\sigma=\sigma_{0}+k_{\mathrm{g}} \bar{L}^{-1 / 2}$

where $\sigma_{0}$ and $k_{\mathrm{g}}$ are the Hall-Petch constants, and $\sigma$ is the yield stress at a mean linear intercept grain size $\bar{L}$. Clearly, such an expression provides a convenient means for developing high strength materials by grain size refinement. Following the first report of grain boundary weakening in nanocrystals [12], there have been numerous studies on the topic and a wide variety of experimental results have been reported, including the continuation of Hall-Petch strengthening, a reduction in the rate of strengthening in the nanocrystalline regime, and grain boundary softening $[13,14]$. While it is recognized that processing and other experimental artifacts can lead to spurious results [15], computer simulations as well as analyses of dislocation processes suggest that below a critical grain size of about $10 \mathrm{~nm}$ grain boundary softening processes will become dominant [16-18]. Grain boundary softening has been attributed to Coble diffusion creep [12], grain boundary shear [19,20] and a reduction in shear modulus [21]. In the present approach, grain boundary softening by Coble creep is considered because the
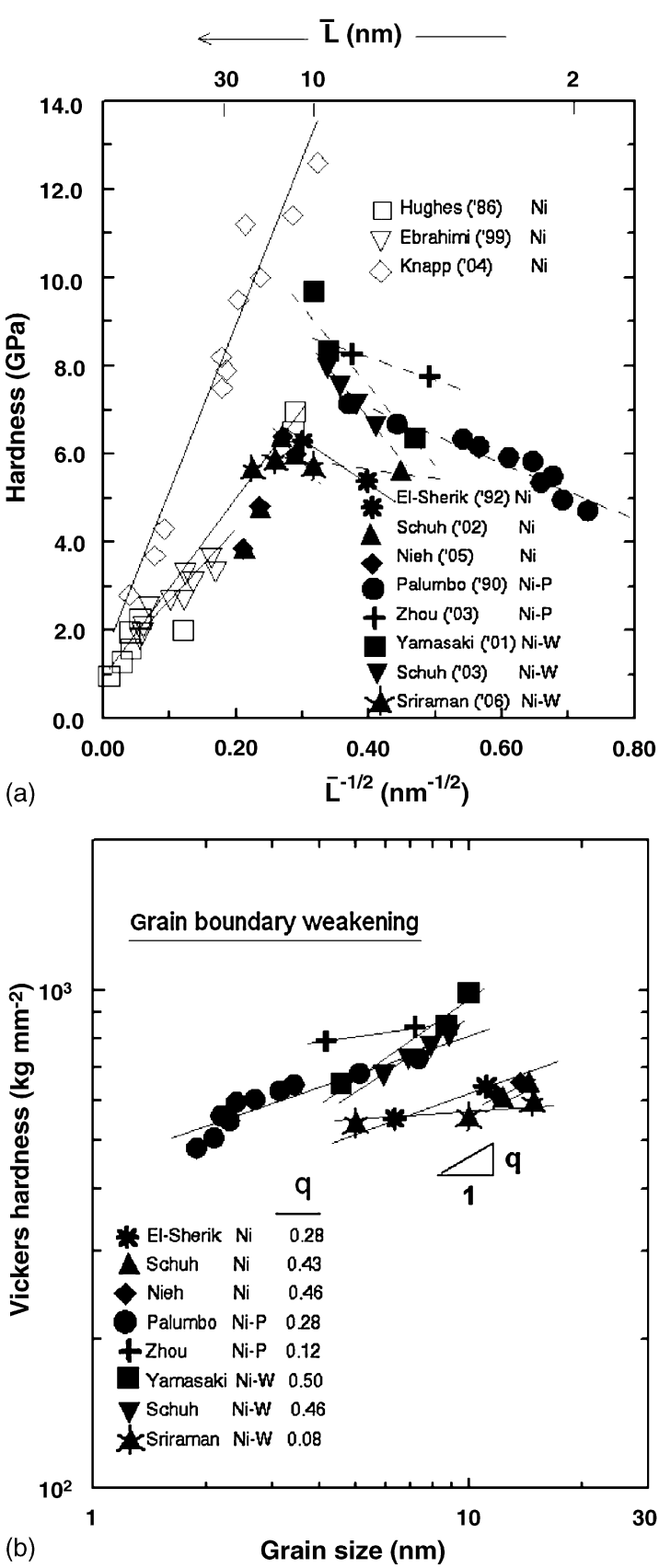

Fig. 3. (a) Conventional Hall-Petch plot for Ni-based materials depicting grain boundary strengthening and weakening. (b) Logarithmic plot of variation in hardness with grain size for materials exhibiting grain boundary weakening in electrodeposited nanocrystalline Ni-based materials.

rate equation is well established theoretically [22]:

$\dot{\varepsilon}_{\mathrm{co}}=\frac{A_{\mathrm{co}} D_{\mathrm{gb}} G b}{k T}\left(\frac{b}{\bar{L}}\right)^{3}\left(\frac{\sigma}{G}\right)$

where the subscript co refers to Coble creep, the constant $A_{\mathrm{co}}=33$, and $D_{\mathrm{gb}}$ is the grain boundary diffusivity.

Fig. 3a displays some experimental results from nanocrystalline Ni-based materials in a conventional linear Hall-Petch format [23-33]; it is clear that there is a wide range in behavior in the nanocrystalline regime, including grain boundary softening. In light of limitations in producing bulk nanomaterials, 
Table 1

Experimental characterization of grain size distributions in nanocrystals

\begin{tabular}{|c|c|c|c|c|c|c|}
\hline S. no. & Material & Synthesis & Arithmetic mean, $\bar{L}(\mathrm{~nm})$ & Median $L_{\mathrm{M}}(\mathrm{nm})$ & Standard deviation $\left(S_{\mathrm{n}}\right)$ & Reference \\
\hline 1 & $\mathrm{Cu}$ & Slide wear & 18.5 & 16.8 & 0.44 & {$[37]$} \\
\hline 2 & $\mathrm{Cu}$ & Slide wear & 49.1 & 42.5 & 0.54 & {$[37]$} \\
\hline 3 & $\mathrm{Ni}$ & Electrodeposition & 11.8 & 10.1 & 0.55 & [26] \\
\hline 4 & $\mathrm{Fe}$ & Ball milling & 6.9 & 6.5 & 0.33 & {$[38]$} \\
\hline 5 & $\mathrm{Cu}$ & SPD & 110.0 & 72.5 & 0.49 & [39] \\
\hline 6 & $\mathrm{Ni}$ & Electrodeposition & 12.3 & 10.5 & 0.56 & {$[40]$} \\
\hline 7 & $\mathrm{Ni}$ & Electrodeposition & 25.9 & 19.7 & 0.54 & [24] \\
\hline 8 & $\mathrm{Co}$ & Electrodeposition & 20.0 & 15.0 & 0.50 & [41] \\
\hline 9 & $\mathrm{Co}$ & Electrodeposition and annealed & 120.0 & 93.0 & 0.42 & {$[41]$} \\
\hline 10 & $\mathrm{Ni}$ & Electrodeposition & 21.4 & 18.0 & 0.35 & {$[42]$} \\
\hline 11 & $\mathrm{Ni}$ & Electrodeposition & 20.7 & 16.0 & 0.38 & {$[42]$} \\
\hline 12 & $\mathrm{Co}$ & Electrodeposition & 11.5 & 11.0 & 0.30 & [43] \\
\hline 13 & $\mathrm{Ni}$ & Laserdeposition & 8.9 & 8.0 & 0.46 & [44] \\
\hline
\end{tabular}

many studies have used hardness $H$ for characterizing strength, with a general assumption that $H=3 \sigma$. The experimental results depicting grain boundary softening are re-plotted in Fig. $3 \mathrm{~b}$ in a logarithmic format of $H$ versus $\bar{L}$. Such experimental data can be expressed as

$H \propto(\bar{L})^{q}$

where $q$ is the slope of the plots shown in Fig. 3b. The experimental results shown in Fig. $3 \mathrm{~b}$ lead to $q$ values between $\sim 0.1$ and 0.5, although Coble creep softening should lead to $q=3$. In addition, it is to be noted that for a given grain size, there is a substantial variation in the reported strengths.

While there have been several studies that explore the effect of grain size distribution on Hall-Petch strengthening and also the transitions in deformation mechanisms in nanocrystals [34-36], there has been no detailed examination of this effect in terms of experimental results shown in Fig. 3b and Eq. (5). The following sections develop an analysis to include the influence of grain size distributions on both grain boundary strengthening and weakening. It is demonstrated that the transition from strengthening to weakening is sensitive to the standard deviation of the grain size distribution, as is the absolute magnitude of the flow stress and the optimum stress.

\subsection{Log-normal grain size distributions in nanocrystals}

A log-normal grain size probability distribution function can be expressed as

$f(L)=\frac{1}{\sqrt{2 \pi} L S_{\mathrm{n}}} \exp \left[-\frac{1}{2}\left(\frac{\ln \left(L / L_{\mathrm{M}}\right)}{S_{\mathrm{n}}}\right)^{2}\right]$

where $L_{\mathrm{M}}$ is the median and $S_{\mathrm{n}}$ is the standard deviation in a number-weighted grain size distribution. For a grain size distribution based on volume instead of numbers, the volume weighted standard deviation $S_{\mathrm{v}}=3 S_{\mathrm{n}}$. The mean grain size $\bar{L}$ can be related to the median value $L_{\mathrm{M}}[36]$

$\bar{L}=L_{\mathrm{M}} \exp \left(\frac{1}{2} S_{\mathrm{n}}^{2}\right)$
Experimental data on grain size distributions in nanocrystalline materials are listed in Table 1, and some of the data are illustrated in Fig. 4 in terms of the probability versus the linear intercept grain size normalized by the mean value [24,26,37-44]. In such plots, all data have to go through the point $L / L_{\mathrm{M}}=1$ at a probability of 50\%. Inspection of Table 1 indicates that typical values of $S_{\mathrm{n}}$ are in the range of $\sim 0.3$ to 0.5 .

\subsection{Grain boundary strengthening and weakening}

The influence of grain size distribution on Hall-Petch strengthening can be examined using the approach developed by Kurzydlowski [45], who assumed that a polycrystal can be assumed to be a composite of groups of grains with a constant size. Under such conditions, for applications to nanocrystalline regime where $\sigma_{0}$ can be neglected, the strength for a constant grain size group $\sigma_{\mathrm{cgs}}^{\mathrm{HP}}$ can be written as

$\sigma_{\mathrm{cgs}}^{\mathrm{HP}}=k_{\mathrm{cgs}} L^{-1 / 2}$

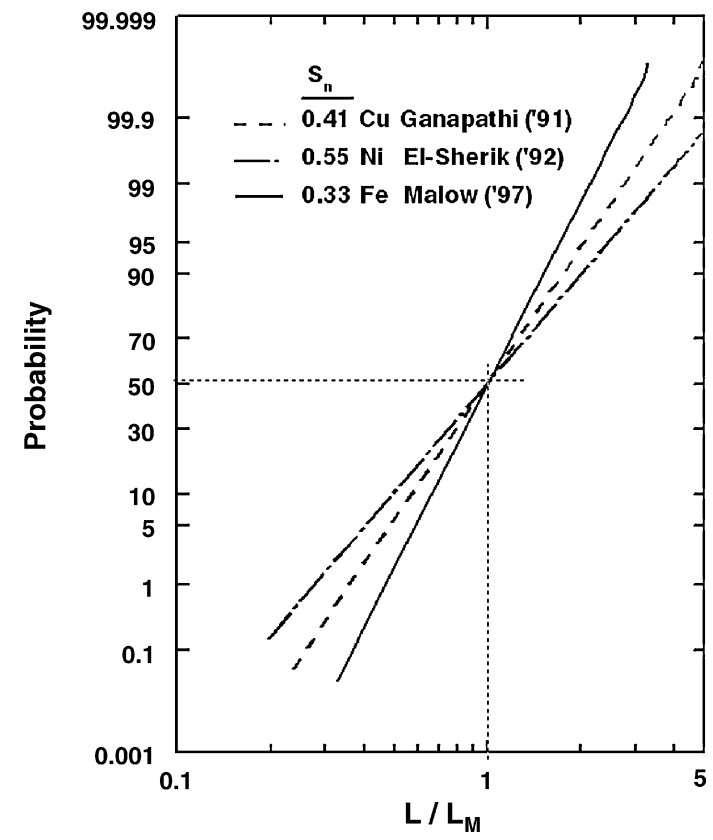

Fig. 4. Grain size distributions in some typical nanocrystalline metals. 
The strength of polycrystals can be calculated, assuming isostrain approximation, as the sum of the volume weighted strengths of the constant grain size groups:

$\sigma^{\mathrm{HP}}=\frac{1}{m_{\mathrm{V}}} \int_{0}^{\infty} V \sigma_{\text {cgs }}^{\mathrm{HP}} f(L) \mathrm{d} L$

where $V$ is the grain volume $\left(=\varphi L^{3}\right.$, with the shape factor $\varphi$ assumed to be $=1$ ). The mean volume of the polycrystal $m_{\mathrm{V}}$ is:

$m_{\mathrm{V}}=\int_{0}^{\infty} L^{3} f(L) \mathrm{d} L$

The analytical solution for the mean volume of polycrystal is

$m_{\mathrm{V}}=\left(L_{\mathrm{M}}\right)^{3} \exp \left(\frac{9}{2} S_{\mathrm{n}}^{2}\right)$

and for the strength of polycrystal is

$\sigma^{\mathrm{HP}}=\frac{k_{\mathrm{cgs}}\left(L_{\mathrm{M}}\right)^{5 / 2} \exp \left((25 / 8) S_{\mathrm{n}}^{2}\right)}{m_{\mathrm{V}}}$

Putting Eqs. (7) and (11) into (12)

$\sigma^{\mathrm{HP}}=k_{\mathrm{cgs}}(\bar{L})^{-1 / 2} \exp \left(-\frac{9}{8} S_{\mathrm{n}}^{2}\right)$

The influence of the standard deviation on the Hall-Petch strengthening slope $k_{\mathrm{g}}$ may be obtained by comparing Eqs. (3) and (13)

$k_{\mathrm{g}}=k_{\mathrm{cgs}} \exp \left(-\frac{9}{8} S_{\mathrm{n}}^{2}\right)$

The above expression is slightly different from that reported by Kurzydlowski [45], with $S_{\mathrm{v}}=3 S_{\mathrm{n}}$, because of the incorporation of Eq. (7) in the present analysis for relating the average linear intercept grain size and the median grain size.

The reduction in flow stress due to Coble grain boundary diffusion creep can be examined based on Eq. (4), for a constant strain rate and temperature:

$\sigma^{\text {Coble }}=B L^{3}$

where

$B=\frac{\dot{\varepsilon} k T}{A_{\mathrm{co}} b D_{\mathrm{gb}}}$

Following the approach taken for Hall-Petch strengthening, the Coble stress for $S_{\mathrm{n}}=0$ can be written as:

$\sigma_{\text {cgs }}^{\text {Coble }}=B_{\text {cgs }} L^{3}$

Then, the strength of polycrystal due to Coble creep for $S_{\mathrm{n}}>0$ can be written as:

$\sigma^{\text {Coble }}=\frac{1}{m_{\mathrm{V}}} \int_{0}^{\infty} L^{3} \sigma_{\mathrm{cgs}}^{\text {Coble }} f(L) \mathrm{d} L$

The analytical solution for Eq. (18) is

$\sigma^{\text {Coble }}=\frac{B_{\mathrm{cgs}}\left(L_{\mathrm{M}}\right)^{6} \exp \left(18 S_{\mathrm{n}}^{2}\right)}{m_{\mathrm{V}}}$

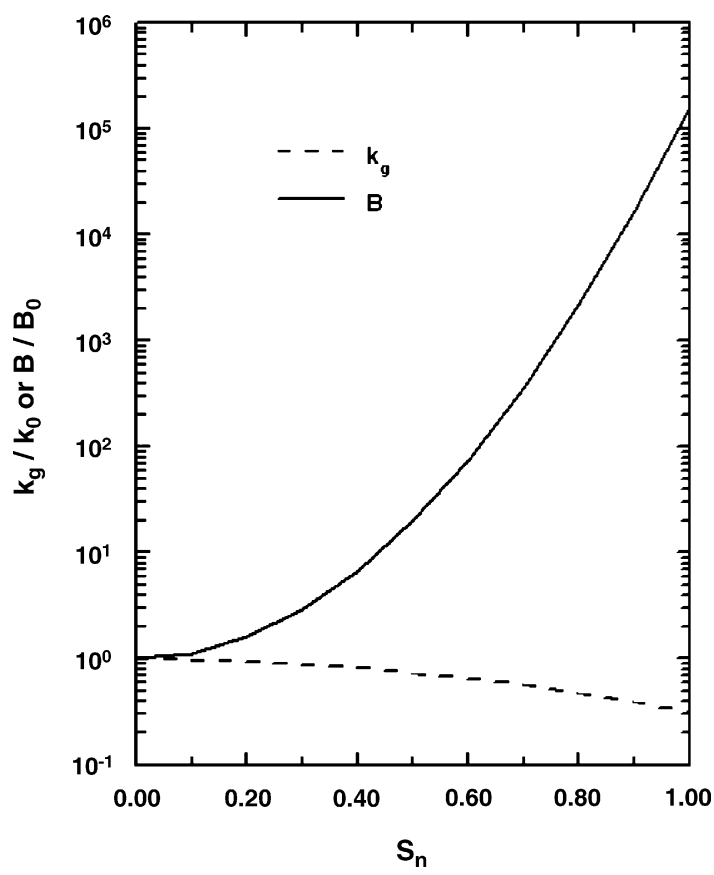

Fig. 5. Influence of the standard deviation of the grain size distributions on the stress associated with Hall-Petch grain boundary strengthening $\left(k_{\mathrm{g}}\right)$ and Coble grain boundary weakening $(B)$.

Putting Eqs. (7) and (11) into Eq. (19):

$\sigma^{\text {Coble }}=B_{\mathrm{cgs}}(\bar{L})^{3} \exp \left(12 S_{\mathrm{n}}^{2}\right)$

Comparing Eqs. (17) and (20)

$B=B_{\mathrm{cgs}} \exp \left(12 S_{\mathrm{n}}^{2}\right)$

It is interesting to note that an essentially identical relationship can be extracted from the analysis by Ter Heege et al. [9]

Fig. 5 illustrates the influence of the grain size distribution on both Hall-Petch strengthening as well as Coble creep weakening in terms of the variation with standard deviation $S_{\mathrm{n}}$ in the constants $k_{\mathrm{g}}$ and $B$; the data are normalized with the values of the constants for $S_{\mathrm{n}}=0$ as $k_{0}$ and $B_{0}$, respectively. Inspection of the figure shows that grain boundary weakening is influenced significantly compared with grain boundary strengthening. An increase in the standard deviation leads to a decrease in the stress associated with grain boundary strengthening and an increase in the stress associated with grain boundary weakening.

\subsection{Transition from grain boundary strengthening to grain boundary weakening ${ }^{1}$}

The approach adopted in this analysis is that there are two independent processes for plastic deformation: Hall-Petch

\footnotetext{
${ }^{1}$ It has been suggested that there may be some other process that becomes important at very fine grain sizes, between the Hall-Petch strengthening and grain boundary softening, but in practice such a region occurs in a narrow range of grain sizes between $\sim 8$ and $20 \mathrm{~nm}$ [18], and this is neglected in the present simple analysis.
} 
strengthening and Coble weakening. Based on the grain size dependence for these processes, it is expected that coarse grained materials will deform by Hall-Petch strengthening whereas fine grained materials will deform by Coble creep. The grain size $L^{*}$ for a transition from strengthening to weakening can be obtained by equating the stresses for strengthening and weakening: $\sigma^{\mathrm{HP}}=\sigma^{\text {Coble }}$. From Eqs. (8) and (17),

$k_{\mathrm{cgs}}\left(L^{*}\right)^{-1 / 2}=B_{\mathrm{cgs}}\left(L^{*}\right)^{3}$

It is assumed for any grain size distribution, grains with sizes less than $L^{*}$ will deform by Coble creep whereas coarser grains will deform by the Hall-Petch process. Then, using iso-strain assumption, the strength of a polycrystal with a grain size distribution can be expressed as:

$\sigma=\frac{1}{m_{\mathrm{V}}} \int_{0}^{L^{*}} \sigma_{\mathrm{cgs}}^{\text {Coble }} L^{3} f(L) \mathrm{d} L+\frac{1}{m_{\mathrm{V}}} \int_{L^{*}}^{\infty} \sigma_{\mathrm{cgs}}^{\mathrm{HP}} L^{3} f(L) \mathrm{d} L$

It is possible to solve this equation analytically using Mathematica 4.1.

\subsection{Analytical results and discussion}

It is necessary to put in values of the constants $B$ and $k_{\mathrm{g}}$ for grain boundary softening and strengthening, respectively, to develop further analytical results. Based on computer simulations and analysis of dislocation processes [16-18], the role of grain size distribution in nanomaterials is examined with the assumptions that for $S_{\mathrm{n}}=0$, the critical grain size for a transition from strengthening to weakening $L^{*}=7 \mathrm{~nm}$ and the optimum strength is $1 \mathrm{GPa}$. These two assumptions essentially fix the values of $B$ and $k_{\mathrm{g}}$ for $S_{\mathrm{n}}=0$, to be used further in Eqs. (14) and (21).

Fig. 6a illustrates the variation in strength with grain size on a logarithmic scale for values of $S_{\mathrm{n}}$ ranging from 0 to 1 . Inspection of the results reveals that an increase in $S_{\mathrm{n}}$ has three important implications: (a) there is a decrease in the grain size for a transition from strengthening to weakening, (b) the optimum strength is reduced, and (c) the transition occurs over a broader range of grain sizes. The slopes of the curves in Fig. 6a give the value of the exponent $q$. The variation in $q$ with grain size is depicted in Fig. 6b, with the grain size being normalized by the critical grain size $L^{*}$ for ease of comparison; in such a plot, all the curves need to pass through a point where $q=0$ and $\bar{L} / L^{*}=1$. As expected, for sufficiently coarse and fine grain sizes, the respective values of $q$ are -0.5 and 3 . However, for a large value of $S_{\mathrm{n}}$, the results indicate that it may not be possible to get into the Coble creep softening regime with $q=3$ regime at realistic grain sizes. Typically, experimental measurements of grain sizes involve errors of $\sim 10 \%$. Then, for experimental values of $S_{\mathrm{n}}$ ranging from 0.3 to 0.5 and values of $\bar{L} / L^{*}$ ranging from 0.9 to 1.1 , the analysis in Fig. $6 \mathrm{~b}$ indicates that the expected $q$ values range from a minimum of -0.3 to a maximum of 0.5 . Thus, a wide range of experimental observations may be possible under nominally similar conditions, including Hall-Petch strengthening with a reduced slope,
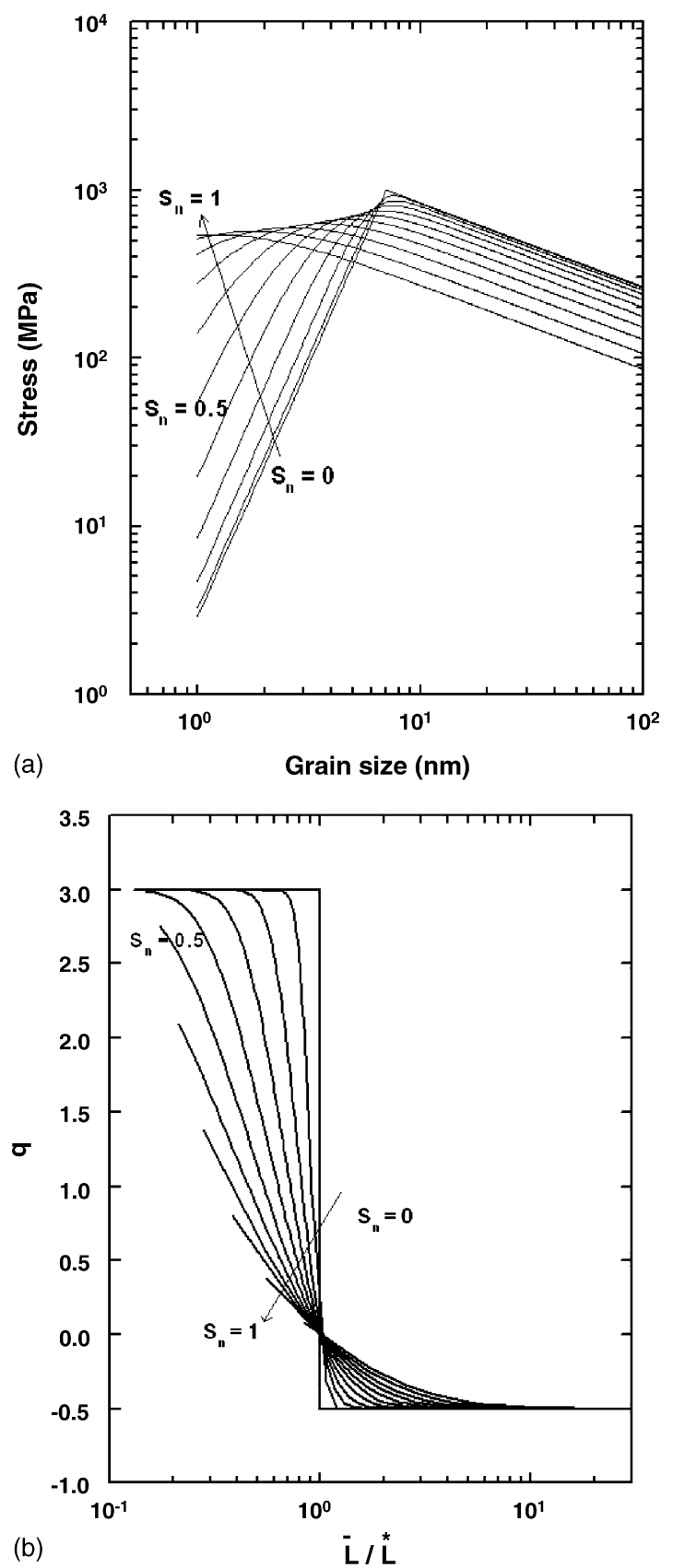

Fig. 6. (a) Influence of the standard deviation of the grain size distribution on the transition from grain boundary strengthening to grain boundary weakening. (b) Influence of the standard deviation of the grain size distribution on the grain size exponent $q$ with the normalized grain size.

a plateau in grain boundary strengthening and grain boundary weakening.

The practical implication of the present analysis is that with typical errors in grain size measurements, there is always a possibility of a difference in experimental observations in the vicinity of a transition from strengthening to softening, for materials with a grain size distribution. Therefore, there is clearly a need to explicitly measure grain size distributions not only in the asprocessed condition but also in the annealed condition because of a possibility of abnormal grain growth in some nanometals [46]. To-date, there appears to have been only one study in which 
the variation in grain size distribution during grain growth has been measured explicitly to establish the occurrence of normal grain growth [37].

It is important to emphasize that although the present analysis considered Coble creep as the relevant grain boundary weakening process, in principle any other weakening process such as grain boundary sliding can also be considered as long as the appropriate rate equation is well known. Irrespective of the details of the grain boundary strengthening and weakening processes, the general concept that the transition will spread out with an increase in the standard deviation is expected to be valid.

\section{Summary and conclusions}

Realistic microstructures of polycrystalline and nanocrystalline materials consist of a log-normal distribution of grain sizes, which has not been considered in most analyses of mechanical properties. Experimental observations are discussed relating to the influence of grain size distributions in superplastic deformation in metals, creep cavitation in ceramics and plastic flow in geological materials. An analysis is developed to consider the influence of grain size distributions on the transition from grain boundary strengthening to grain boundary softening in nanocrystalline metals. The transition from grain boundary hardening to softening becomes broader with an increase in the grain size distribution. It is demonstrated that a wide range of experimental behavior can be expected in nanocrystals, with the experimentally observed grain size distributions and usual errors in grain size measurements.

\section{Acknowledgments}

This work was supported by the Department of Science and Technology. Additional support from a General Motors collaborative research program is also acknowledged gratefully.

\section{References}

[1] A.K. Mukherjee, J.E. Bird, J.E. Dorn, Trans. ASM 62 (1969),

[2] J.E. Bird, A.K. Mukherjee, J.E. Dorn, in: D.G. Brandon, A. Rosen (Eds.), Quantitative Relation Between Properties and Microstructure, Israel Univ. Press, Jerusalem, 1969, p. 255.

[3] A.H. Chokshi, A.K. Mukherjee, T.G. Langdon, Mater. Sci. Eng. R 10 (1993) 237-274.

[4] R. Raj, A.K. Ghosh, Acta Metall. 29 (1981) 283-292.

[5] A.H. Chokshi, A.K. Mukherjee, Metall. Trans. A 19 (1988) 1621-1624.

[6] J. Bai, R. Raj, Metall. Mater. Trans. A 36 (2005) 2913-2919.

[7] A.H. Chokshi, J.R. Porter, J. Am. Ceram. Soc. 70 (1987) 197-202.

[8] R. Heilbronner, D. Bruhn, J. Struct. Geol. 20 (1998) 695-705.

[9] J.H. Ter Heege, J.H.P. De Bresser, C.J. Spiers, J. Struct. Geol. 26 (2004) 1693-1705.
[10] E.O. Hall, Proc. Phys. Soc. B 64 (1951) 747-753.

[11] N.J. Petch, J. Iron Steel Inst. 174 (1953) 25-28.

[12] A.H. Chokshi, A. Rosen, J. Karch, H. Gleiter, Scripta Metall. 23 (1989) 1679-1684.

[13] D.G. Morris, in: Dinesen, et al. (Eds.), Proceedings of the 22nd Riso Int. Conf. on Mater. Sci.: Sci. Metastable and Nanocrystalline Alloys-Structure, Properties and Modeling, 2001, pp. 89-104.

[14] M.A. Meyers, A. Mishra, D.J. Benson, Prog. Mater. Sci. 51 (2006) $427-$ 556.

[15] S.R. Agnew, B.R. Elliott, C.J. Youngdahl, K.J. Hemker, J.R. Weertman, Mater. Sci. Eng. A 285 (2000) 391-396.

[16] J. Schiotz, F.D. Di Tolla, K.W. Jacobsen, Nature 391 (1998) 561-563.

[17] H. Van Swygenhoven, A. Caro, D. Farkas, Scripta Mater. 44 (2001) $1513-1516$.

[18] R.J. Asaro, S. Suresh, Acta Mater. 53 (2005) 3369-3382.

[19] H. Hahn, K.A. Padmanabhan, Phil. Mag. 76B (1997) 559-571.

[20] H. Conrad, J. Narayan, Scripta Mater. 42 (2000) 1025-1030.

[21] N. Wang, Z. Wang, K.T. Aust, U. Erb, Acta Metall. Mater. 43 (1995) 519-528.

[22] R.L. Coble, J. Appl. Phys. 34 (1963) 1679-1682.

[23] G.D. Hughes, S.D. Smith, C.S. Pande, H.R. Johnson, R.W. Armstrong, Scripta Metall. 20 (1986) 93-97.

[24] F. Ebrahimi, Z. Ahmed, L.M. Kristin, Mater. Res. Soc. Symp. Proc. 634 (B2.7) (2001) 1-6.

[25] J.A. Knapp, D.M. Follstaedt, J. Mater. Res. 19 (2004) 218-227.

[26] A.M. El Sherik, U. Erb, G. Palumbo, K.T. Aust, Scripta Metall. Mater. 27 (1992) 1185-1188.

[27] C.A. Schuh, T.G. Nieh, T. Yamasaki, Scripta Mater. 46 (2002) 735-740.

[28] T.G. Nieh, J.G. Wang, Intermetallics 13 (2005) 377-385.

[29] G. Palumbo, U. Erb, K.T. Aust, Scripta Metall. Mater. 24 (1990) 2347-2350.

[30] Y. Zhou, U. Erb, K.T. Aust, G. Palumbo, Scripta Mater. 48 (2003) 825-830.

[31] T. Yamasaki, Scripta Mater. 44 (2001) 1497-1502.

[32] C.A. Schuh, T.G. Nieh, H. Iwasaki, Acta Mater. 51 (2003) 431-443.

[33] K.R. Sriraman, S.G. Raman, S.K. Seshadri, Mater. Sci. Eng. 418 (2006) 303-311.

[34] R.A. Masumara, P.M. Hazzledine, C.S. Pande, Acta Mater. 46 (1998) 4527-4534.

[35] T. Morita, R. Mitra, J.R. Weertman, Mater. Trans. 45 (2004) 502-508.

[36] B. Zhu, R.J. Asarao, P. Krysl, R. Bailey, Acta Mater. 53 (2005) 4825-4838.

[37] S.K. Ganapathi, D.M. Owen, A.H. Chokshi, Scripta Metall. Mater. 25 (1991) 2699-2704.

[38] T.R. Malow, C.C. Koch, Acta Mater. 45 (1997) 2177-2186.

[39] R.K. Islamgaliev, F. Chmelik, R. Kuzel, Mater. Sci. Eng. A 237 (1997) 43-52.

[40] D.H. Jeong, F. Gonzalez, G. Palumbo, K.T. Aust, U. Erb, Scripta Mater. 44 (2001) 493-499.

[41] G. Hibbard, K.T. Aust, G. Palumbo, U. Erb, Scripta Mater. 44 (2001) 513-518.

[42] F. Dalla Torre, Van Swygenhoven, M. Victoria, Acta Mater. 50 (2002) 3957-3970.

[43] A.A. Karimpoor, U. Erb, K.T. Aust, G. Palumbo, Scripta Mater. 49 (2003) 651-656.

[44] Z. Shan, E.A. Stach, J.M.K. Wiezorek, J.A. Knapp, D.M. Folltaedt, S.X. Mao, Science 305 (2004) 654-657.

[45] K.J. Kurzydlowski, Scripta Metall. Mater. 24 (1990) 879-883.

[46] M. Thuvander, M. Abraham, A. Cerezo, G.D.W. Smith, Mater. Sci. Technol. 17 (2001) 961-970. 